\title{
SHAMS: Combining chemical modification of RNA with mass spectrometry to examine polypurine tract-containing RNA/DNA hybrids
}

\author{
KEVIN B. TURNER, ${ }^{1}$ HYE YOUNG YI-BRUNOZZI, ${ }^{2}$ ROBERT G. BRINSON, ${ }^{2}$ JOHN P. MARINO, ${ }^{3}$ \\ DANIELE FABRIS, ${ }^{1}$ and STUART F.J. LE GRICE ${ }^{2}$ \\ ${ }^{1}$ Department of Chemistry and Biochemistry, University of Maryland Baltimore County, Baltimore, Maryland 21250, USA \\ ${ }^{2}$ RT Biochemistry Section, HIV Drug Resistance Program, National Cancer Institute, National Institutes of Health, Frederick, \\ Maryland 21702, USA \\ ${ }^{3}$ Center for Advanced Research in Biotechnology, University of Maryland Biotechnology Institute and National Institute of Standards \\ and Technology, Rockville, Maryland 20850, USA
}

\begin{abstract}
Selective 2'-hydroxyl acylation analyzed by primer extension (SHAPE) has gained popularity as a facile method of examining RNA structure both in vitro and in vivo, exploiting accessibility of the ribose 2 '-OH to acylation by $\mathrm{N}$-methylisatoic anhydride (NMIA) in unpaired or flexible configurations. Subsequent primer extension terminates at the site of chemical modification, and these products are fractionated by high-resolution gel electrophoresis. When applying SHAPE to investigate structural features associated with the wild-type and analog-substituted polypurine tract (PPT)-containing RNA/DNA hybrids, their size (20-25 base pairs) rendered primer extension impractical. As an alternative method of detection, we reasoned that chemical modification could be combined with tandem mass spectrometry, relying on the mass increment of RNA fragments containing the NMIA adduct $\left(M_{r}=133 \mathrm{Da}\right)$. Using this approach, we demonstrate both specific modification of the HIV-1 PPT RNA primer and variations in its acylation pattern induced by replacing template nucleotides with a non-hydrogen-bonding thymine isostere. Our selective 2'-hydroxyl acylation analyzed by mass spectrometry strategy (SHAMS) should find utility when examining the structure of small RNA fragments or RNA/DNA hybrids where primer extension cannot be performed.
\end{abstract}

Keywords: retroviruses; polypurine tract; RNA/DNA hybrid; chemical modification; mass spectrometry; NMR spectroscopy

\section{INTRODUCTION}

Plus strand, DNA-dependent DNA synthesis in retroviruses and long terminal repeat (LTR)-containing retrotransposons initiates from a purine-rich segment of the viral RNA genome designated the polypurine tract (PPT) (Klarmann et al. 2002; Rausch and Le Grice 2004). Subsequent PPT removal from nascent DNA is critical to creating the appropriate 5' LTR terminus for recognition by the viral integration machinery. While the accuracy with which the primer terminus is created from the RNA/DNA replication intermediate and the PPT is later excised from nascent

Reprint requests to: Stuart F.J. Le Grice, RT Biochemistry Section, HIV Drug Resistance Program, National Cancer Institute, National Institutes of Health, Frederick, MD 21702, USA; e-mail: legrices@mail.nih.gov; fax: (301) 846-6013.

Article published online ahead of print. Article and publication date are at http://www.rnajournal.org/cgi/doi/10.1261/rna.1615409.
DNA can be accurately reproduced in vitro (Rausch and Le Grice 1997), the structural basis for its selection remains unclear. Recent single-molecule studies have indicated that dynamic binding orientations may direct the DNA polymerase and ribonuclease $\mathrm{H}$ (RNase $\mathrm{H}$ ) activities of human immunodeficiency virus type 1 reverse transcriptase (HIV-1 RT), i.e., the composition of the PPT primer dictates whether enzyme favors its polymerization- or RNase H-competent state (Abbondanzieri et al. 2008).

The possibility that such complex protein/nucleic acid interplay might exploit unusual structural features of the duplex has been suggested by crystallographic analysis of HIV-1 RT bound to a PPT-containing RNA/DNA hybrid (Sarafianos et al. 2001). This study highlighted a collection of weakened base pairs, unpaired and mispaired bases, collectively designated the "unzipped" region (Fig. 1). However, whether these structural rearrangements result from enzyme binding or reflect an unusual, pre-existing geometry exploited by RT remains to be established. To address 


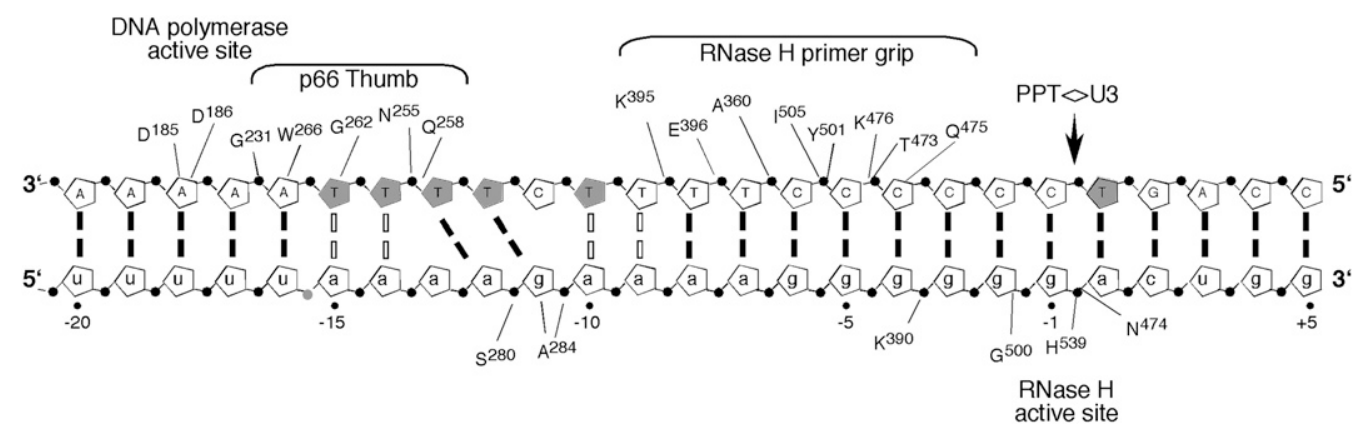

FIGURE 1. Model for disposition of HIV-1 RT on the PPT, showing duplex "unzipping" between -9 and -15 (Sarafianos et al. 2001) and $\mathrm{KMnO}_{4}$-sensitive template thymines (filled pentagon) (Kvaratskhelia et al. 2002). RNase $\mathrm{H}$ primer grip residues of p66 are relocated on the duplex relative to positioning His539 of the catalytic center to the scissile bond, i.e., the PPT-U3 junction. Contacts made by the thumb subdomain and polymerase active site are modeled accordingly and based on crystallographic data of RT complexes with either duplex DNA (Jacobo-Molina et al. 1993; Huang et al. 1998) or RNA/DNA (Sarafianos et al. 2001). DNA and RNA bases are in uppercase and lowercase, respectively.

this experimentally, we have examined thymine sensitivity in the PPT (-) DNA template to $\mathrm{KMnO} 4$ oxidation (Kvaratskhelia et al. 2002), noting that the $(\mathrm{rA})_{4}:(\mathrm{dT})_{4}$ tracts deviated from canonical Watson-Crick geometry. $\mathrm{KMnO}_{4}$ sensitivity of a template thymine immediately adjacent to the PPT/U3 junction also suggested that altered duplex geometry at the scissile bond might contribute to RNase $\mathrm{H}$ cleavage specificity.

Here, we focused our attention on structural features of the RNA primer of the HIV-1 PPT RNA/DNA hybrid, and report a new variation of the selective 2 '-hydroxyl acylation analyzed by primer extension (SHAPE) footprinting strategy (Wilkinson et al. 2006) that interrogates accessibility of the ribose $2^{\prime}-\mathrm{OH}$ group to $N$-methylisatoic anhydride (NMIA). In flexible regions, such as loops, bulges, and junctions, the ribose pucker adopts a conformation allowing formation of a nucleophilic $2^{\prime}$-oxyanion that reacts with NMIA to form a bulky $2^{\prime}$-O-adduct. Reactivity of this functional group is inhibited when the nucleotide is either included in folded helical structures or protected by interactions with cognate proteins and other ligands. Primer extension subsequently creates a cDNA library corresponding to termination at sites of adduct formation. While SHAPE has elucidated two-dimensional structures of several regulatory RNAs (Badorrek and Weeks 2006; Wilkinson et al. 2006; Vicens et al. 2007; Gherghe et al. 2008; Wang et al. 2008; Wilkinson et al. 2008), there are no reports on its use to study the architecture of RNA in the context of RNA/DNA hybrids. Moreover, the size and purine-rich sequence of wild-type and analog-substituted RNA/DNA hybrids (20-25 base pairs [bp]) made the primer extension step impractical. To probe the PPT RNA primer strand using selective $2^{\prime}$-hydroxyl acylation, we therefore combined chemical modification with electrospray ionization-Fourier transform ion cyclotron resonance (ESI-FTICR) (Comisarow and Marshall 1974; Hendrickson et al. 1999) mass spectrometry to identify sites of modification directly through the mass increment of fragmented RNA containing the 133.05-Da NMIA adduct. Using this approach, we uncovered NMIA-sensitive positions within the PPT primer whose sensitivity responds to introducing the non-hydrogen-bonding thymine isostere 2,4-difluoro-5-methylbenzene (dF) (Guckian et al. 1998) into the (-) DNA template. Lack of reactivity at equivalent sites of a Saccharomyces cerevisiae retrotransposon Ty3 PPT indicates that these structural anomalies are a unique signature of the HIV plus strand primer, possibly indicating coevolution of the retroviral polymerase and its cognate PPT. This simple variation of SHAPE should have general utility when analyzing short RNAs and RNAs whose sequence prevents primer extension.

\section{RESULTS}

\section{Experimental rationale}

Unlike traditional chemical or enzymatic approaches for probing RNA structure (Ehresmann et al. 1987), SHAPE is particularly advantageous in that modification is baseindependent, i.e., all ribonucleotides are probed in a single reaction. Whereas previous chemical probes can differentiate single- versus double-stranded regions by targeting the base-pairing face of individual nucleotides, NMIA reports on the flexibility of the phosphodiester backbone by reacting with the $2^{\prime}-\mathrm{OH}$ group of nucleotides that are not involved in canonical Watson-Crick pairing or protected by ligand interactions. Our interest in combining NMIA treatment with mass spectrometric detection was prompted by the need to examine the RNA component of short (20$25 \mathrm{bp}$ ), synthetic RNA/DNA hybrids on which primer extension was impractical. Since ribose $2^{\prime}-\mathrm{OH}$ acylation is accompanied by a 133.05 -Da mass increment corresponding to the NMIA adduct (Wilkinson et al. 2006), we predicted that the position of modification could be determined on the basis of characteristic fragmentation patterns derived by tandem mass spectrometry. 


\section{Selective 2'-hydroxyl acylation analyzed by mass spectrometry (SHAMS)}

An important concern in chemical probing of nucleic acids is the possibility that initial modification of exposed sites induces conformational changes of the target structure, exposing additional sites that were originally protected. The resulting modification pattern would therefore not provide an accurate representation of the native structure, but include significant unwarranted deviations. Subtle variations of $\mathrm{pH}$ and ionic strength associated with the probing reaction can also contribute to distorting the substrate conformation, potentially leading to erroneous and artifactual information. These concerns can be minimized by performing chemical probing under "single-hit" conditions by judiciously selecting the probe:substrate ratio. Unfortunately, the leading analytical platforms make verifying fulfillment of single-hit conditions problematic. Primer extension, for example, relies on the ability of NMIA modification to halt DNA polymerization by reverse transcriptase, thus producing oligonucleotides of specific length determined by the adduct position. However, when the primers are targeted only to the substrate ends, polymerization will effectively stop at the first adduct encountered, while any modifications existing downstream on the same molecule will remain undetected.

Chemical probes can be employed with direct mass spectrometric detection, due to unique mass signatures that enable immediate characterization of their stoichiometry and sequence position ( $\mathrm{Yu}$ and Fabris 2003, 2004; Kellersberger et al. 2004). The flexibility afforded by this platform allows utilization of both mono- and bifunctional probes, which are critical for revealing long-range interactions and spatial relationships necessary to model complex three-dimensional structures (Yu et al. 2005, 2008). More significantly, the ability to directly monitor the number of modified sites provides the basis for an effective strategy for recognizing the occurrence of probing distortion, which consists of titrating the target structure with increasing amounts of probe in individual reactions. In this way, the onset of possible distortion and unfolding can be readily recognized by the detection of new sites that become reactive in the titration series.

This approach is exemplified by NMIA probing of the PPT RNA/DNA hybrid and direct monitoring by ESIFTICR. As shown by the spectrum obtained in a 10 -fold NMIA excess, up to three NMIA adducts are clearly recognizable from their characteristic incremental mass of 133.05 Da, together with a significant proportion of unmodified RNA (Fig. 2A). The complementary DNA strand is also observed, due to melting of the RNA/DNA hybrid before MS analysis. All species display the sodium adducts typically observed in nucleic acid analysis (Turner et al. 2008b). As the NMIA concentration was progressively increased, adduct intensity increased and that of unmod-
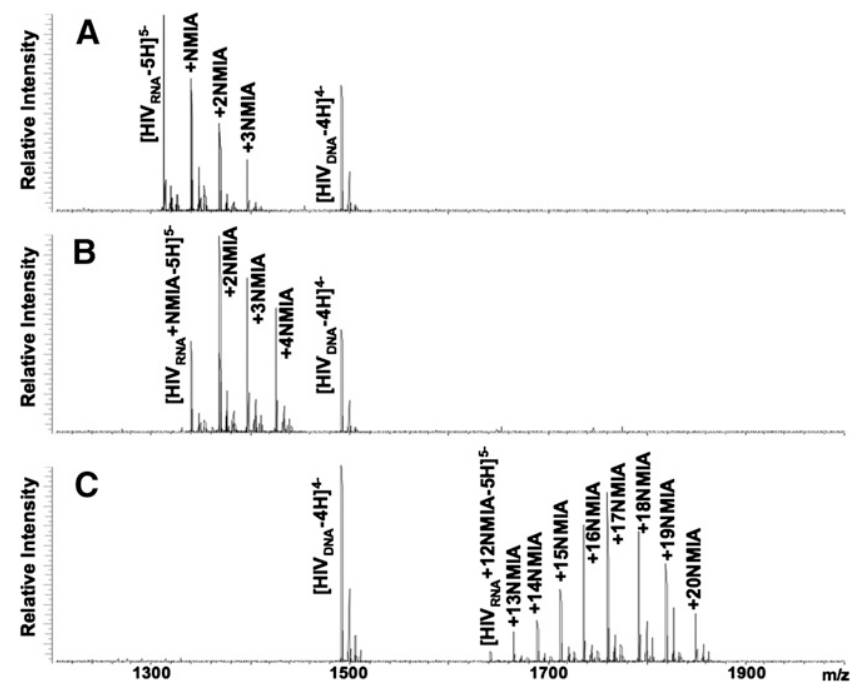

FIGURE 2. Nano-ESI mass spectra of HIV-1 PPT hybrid after reaction with a $(A)$ 10-fold, $(B)$ 50-fold, and $(C)$ 100-fold NMIA excess for $50 \mathrm{~min}$ at $37^{\circ} \mathrm{C}$. Under minimal reaction conditions, the majority of the RNA substrate remains unmodified, while in the bottom spectrum the entire RNA substrate is modified. In both cases, the DNA strand is not modified by NMIA owing to the absence of a ribose $2^{\prime}-\mathrm{OH}$ group.

ified RNA decreased correspondingly (data not shown). However, new adducts were only noted at a 50-fold NMIA excess, concomitant with which was the loss of all unmodified substrate (Fig. 2B). The number of adducts increased linearly with probe concentration, to the point where all possible $2^{\prime}-\mathrm{OH}$ groups were found to be susceptible to modification by a 100 -fold of reagent (Fig. 2C). Product accumulation observed at higher NMIA concentrations, followed by the sudden increase of the number of adducts, suggesting substrate unfolding induced by modification of primary sites in the native hybrid.

Mass spectrometry can, in addition, provide direct information about the relative abundance of the modification products, which can be problematic for other analytical platforms. Indeed, the negative charges necessary for ESI-FTICR detection are carried by the phosphate groups of the oligonucleotide backbone, which are unaffected by ribose modification. This observation is substantiated here by the fact that all RNA species in Figure 2 (modified and unmodified) exhibited the same charge state. Therefore, signal intensities were a direct reflection of the respective abundances in solution, providing a relative scale of reactivity at different sites.

\section{NMIA sensitivity of HIV-1 PPT ribonucleotides}

Confident that the greater reactivity directly reflected structural flexibility, rather than conformational effects induced by initial modifications, we next proceeded to map the position of the sites exposed at the lower NMIA 
concentration of Figure 2A. This was accomplished by sustained off-resonance irradiation-collision-induced dissociation (SORI-CID) (Gauthier et al. 1991), which involves isolating the precursor ion of interest in the FTICR cell, then activating it with appropriate $\mathrm{rf}$ and Ar pulses (see Materials and Methods). Gas-phase dissociation of nucleic acids produces characteristic backbone fragments reflecting the sequence of the precursor ion (McLuckey and HabibiGoudarzi 1993; Nordhoff et al. 1996), exemplified by spectra obtained from reaction mixtures containing NMIA and HIV-1 PPT substrate (Fig. 3). Interpreting this type of data is facilitated by comparing the results from unmodified and modified RNA products. In the case of the former, characteristic c (also described as $\mathrm{d}-\mathrm{H}_{2} \mathrm{O}$ ) and y series ions were

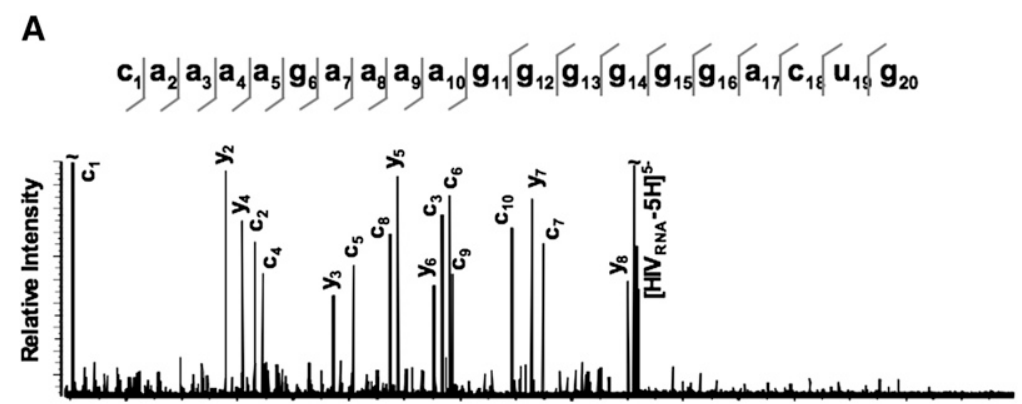

B
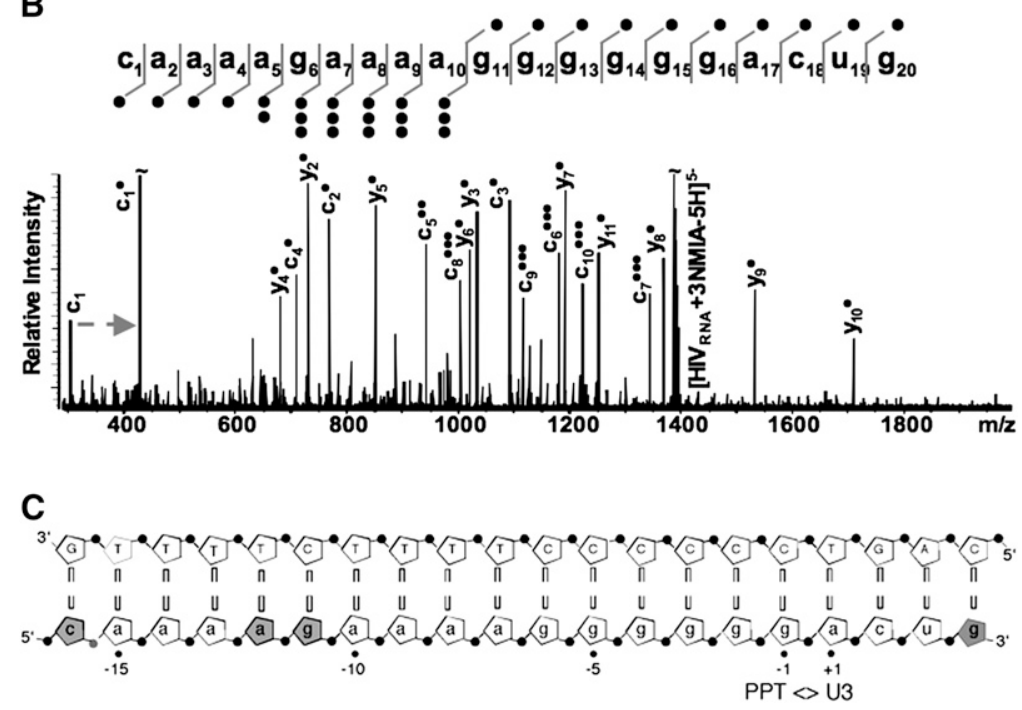

FIGURE 3. (A) SORI-CID spectrum of the unmodified RNA substrate in the -5 charge state. Although all peaks could be assigned, for clarity only the main sequence ions ( $c$ and y ions) were labeled using the standard mass spectrometry notation. Gray lines throughout the sequence correspond to the labeled fragments. (B) SORI-CID spectrum of the triply modified RNA substrate in the -5 charge state. Only the modified fragments were labeled for clarity. Owing to the modification occurring at the $2^{\prime}-\mathrm{OH}$, the $\mathrm{d}-\mathrm{H}_{2} \mathrm{O}$ series cannot undergo the loss of $\mathrm{H}_{2} \mathrm{O}$ and therefore is observed as a $\mathrm{c}$ series. The gray arrow highlights the mass shift of 133.05 Da for one NMIA modification from the unmodified to the modified $c_{1}$ ion. $(\bullet)$ The number of NMIA modifications observed on the corresponding fragment; for example, the $\mathrm{C}_{5} \bullet \bullet$ ion corresponds to the "CAAAA" fragment with two modifications. $(C)$ Schematic of the HIV-1 PPT RNA/DNA hybrid using standard biological numbering as used in the Results and Discussion. Gray pentagons indicate NMIA ribose sensitivity observed by mass spectrometry. readily recognized (Fig. 3A), as typically expected from type of nucleic acid molecule (Cerny et al. 1987; and Schurch 2005). Activation of the triply modified s cies enabled detection of $\mathrm{c}$ ions that were shifted by the characteristic NMIA incremental mass (Fig. 3B), thus revealing the sequence position of the various adducts (Fig.

Inspection of the ion series indicated that the triply modified species comprised distinct product populations NMIA adducts distributed over four different sites . 3C), as confirmed also by SORI-CID spectra obtained shown). Enhanced reactivity of terminal nucleotides reflects fraying of the duplex ends, consistent with our recent NMR studies (Yi-Brunozzi et al. 2008). Acylation of ribonucleotides $-12 \mathrm{a}$ and $-11 \mathrm{~g}$ (Fig. 3C) may be a direct consequence of their mispairing with template nucleotides $-13 \mathrm{~T}$ and $-12 \mathrm{~T}$, respectively, determined from the co-crystal structure of HIV-1 RT bound to its PPT (Sarafianos et al. 2001). In contrast, NMIA reactivity provided no evidence for the unpairing of ribonucleotide $-13 \mathrm{a}$, as suggested by the crystal structure. In this respect, it is important to note that while $\mathrm{KMnO}_{4}$ probing indicated accessibility of template thymines $-15 \mathrm{~T},-14 \mathrm{~T}$, $-13 \mathrm{~T}$, and $-12 \mathrm{~T}$, suggesting deviation from canonical Watson-Crick geometry (Kvaratskhelia et al. 2002), no such anomalies were reported by the NMR analysis that instead confirmed WatsonCrick base-pairing throughout the HIV-1 PPT RNA/DNA hybrid in the absence of RT (Yi-Brunozzi et al. 2008). In particular, no upfield shifting of imino protons was observed, as might be expected based on the $-11 \mathrm{~g} /-12 \mathrm{~T}$ mismatch observed in the crystal structure of Sarafianos et al. (2001).

In light of the NMR observations, one interpretation of the NMIA probing data is that a small population of the HIV-1 PPT is sufficiently malleable to assume an alternative configuration where base-pairing is transiently lost. In support of this, X-ray crystallography of an octanucleotide RNA/DNA hybrid derived from the HIV-1 PPT has shown that anomalous stacking renders the $-12 \mathrm{a} /-11 \mathrm{~g} /-10 \mathrm{a}$ step highly deformable, which may be sufficient to promote 
transient loss of base-pairing (Kopka et al. 2003). Alternatively, $-12 \mathrm{a} /-11 \mathrm{~g}$ NMIA sensitivity may reflect an alteration in sugar pucker from the $\mathrm{C}^{\prime}$-endo to $\mathrm{C} 2{ }^{\prime}$-endo conformation, which has been suggested to mediate ligand recognition (which in this case would be HIV RT) and catalysis (Vicens et al. 2007; Gherghe et al. 2008).

\section{NMIA sensitivity of analog-substituted HIV-1 PPTs}

Previous studies have shown that introducing the nonhydrogen-bonding thymine isostere 2,4-difluoro-5-methylbenzene $(\mathrm{dF})$ at selected positions of the DNA template in the HIV-1 PPT facilitates NMR assignment of many carbon and proton resonances of the PPT substrates while also acting as a subtle probe of hybrid structure (Yi-Brunozzi et al. 2008). dF substitutions within the PPT DNA were also shown to significantly re-direct RNase $\mathrm{H}$ cleavage specificity of the modified RNA/DNA hybrids (Rausch et al. 2003). Based on these combined observations, $\mathrm{dF}$ was substituted for $-13 \mathrm{~T},-8 \mathrm{~T}$, and $+1 \mathrm{~T}$ on the PPT DNA template, and NMIA sensitivity of analog-substituted RNA/DNA hybrids was examined (Fig. 4).

A $-13 \mathrm{~T} / \mathrm{dF}$ substitution resulted in a maximum of four NMIA adducts under the same reaction conditions employed for the wild-type hybrid, which SORI-CID revealed as being produced by the modification of five distinct sites (data not shown). As observed for the wild-type hybrid, terminal ribonucleotides $-16 \mathrm{c}$ and $+4 \mathrm{~g}$, along with internal ribonucleotides $-12 \mathrm{a}$ and $-11 \mathrm{~g}$, were acylated (Fig. $4 \mathrm{~A}$ ). Modification of the additional site corresponding to ribonucleotide -13 a was directly ascribable to elimination of

A

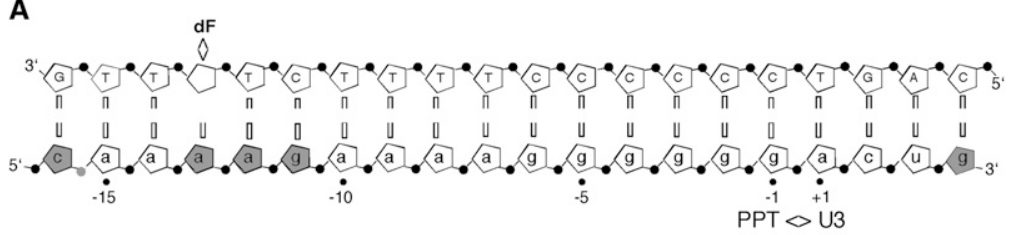

B

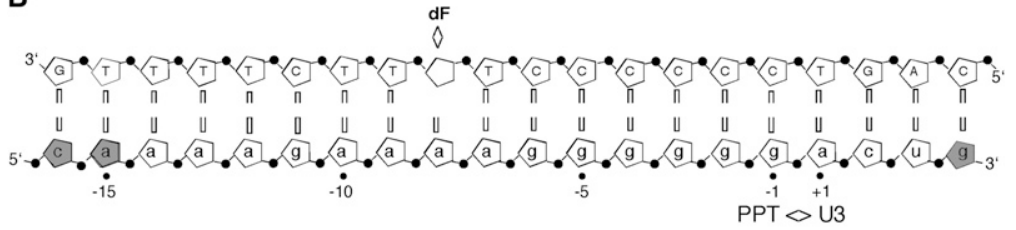

C

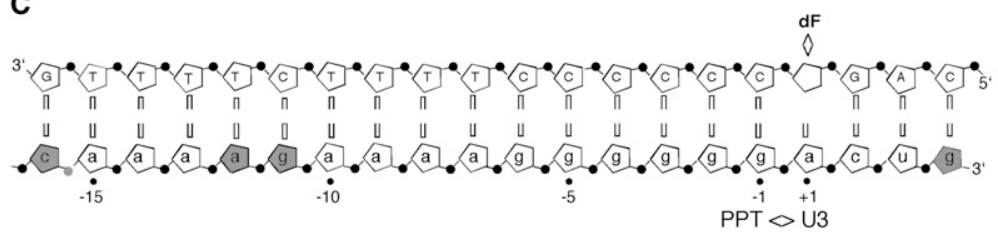

FIGURE 4. Schematic of the HIV-1 PPT RNA/DNA hybrid with (open diamond) T/dF substitutions at template positions $(A)-12,(B)-8$, and $(C)+1$. Gray pentagons indicate positions of NMIA sensitivity observed by mass spectrometry. hydrogen-bonding capacity with $\mathrm{dF}$ (Fig. 4A). In contrast, a $-8 \mathrm{~T} / \mathrm{dF}$ substitution eliminated acylation of PPT ribonucleotides $-11 \mathrm{~g}$ and $-12 \mathrm{a}$ (Fig. 4B). Equally unexpected was the finding that ribonucleotide $-8 \mathrm{a}$ remained insensitive to acylation despite its inability to pair with $-8 \mathrm{dF}$. Finally, a hybrid wherein $\mathrm{dF}$ was substituted for $+1 \mathrm{~T}$ retained reactivity at the internal ribonucleotides $-12 \mathrm{a}$ and $-11 \mathrm{~g}$, but failed to elicit NMIA sensitivity of the +1a RNA complement, analogous to our observations with the $-8 \mathrm{~T} / \mathrm{dF}$ template substitution (Fig. 4C).

Given the unusual acylation patterns of dF-substituted hybrids, ${ }^{19} \mathrm{~F}$-NMR was next used to determine if anomalous structural features could be detected at the sites of analog replacement. What is immediately apparent from the $1 \mathrm{D}$ ${ }^{19} \mathrm{~F}$ spectra of the $-13 \mathrm{~T} / \mathrm{dF}$ and $-8 \mathrm{~T} / \mathrm{dF}$-substituted templates (Fig. 5A,B) is that the modified PPT hybrids exhibited two major and at least two minor peaks for the fluorine resonances associated with the $\mathrm{dF}$ substitution. Since the purity of the substituted DNA strand and RNA:DNA stand stoichiometry were confirmed by mass spectrometry, the presence of the minor peaks suggests a small population of an alternative conformer(s). In addition, while the pyrimidine isostere exists in a $5^{\prime}-\mathrm{T}-\mathrm{T}-\mathrm{dF}-\mathrm{T}$ $\mathrm{C}-3^{\prime}$ sequence context for both the $-13 \mathrm{~T} / \mathrm{dF}-$ and $-8 \mathrm{~T} / \mathrm{dF}-$ substituted templates, the chemical shifts associated with the fluorine resonances of the $\mathrm{dF}$ bases are significantly different, indicating differences in the local stacking of these bases in the RNA/DNA hybrid (Fig. 5A,B). Moreover, NOE correlation patterns observed in HF NOESY experiments acquired on the $-13 \mathrm{~T} / \mathrm{dF}$ - and $-8 \mathrm{~T} / \mathrm{dF}$-substituted templates (data not shown) are consistent with minor groove compression in the central A-tract. These distinguishing observations of altered base-stacking and minor groove compression presumably renders the 2'-hydroxyl inaccessible to acylation with NMIA at position -8 despite the loss of hydrogen-bonding capacity. Furthermore, the ability to alter the acylation profile at a distance through a relatively benign insertion of a thymine isostere indicates an extraordinary sensitivity of the "unzipped" region of the PPT RNA/DNA hybrid to minor conformational changes.

The $1 \mathrm{D}{ }^{19} \mathrm{~F}$ spectrum of the $+1 \mathrm{~T} / \mathrm{dF}-$ substituted RNA/DNA hybrid differs from that of its $-8 \mathrm{~T} / \mathrm{dF}$ - and $-13 \mathrm{~T} /$ dF-substituted counterparts (Fig. 5C) in that no minor peaks are detectable. This indicates a unique environment at the $\mathrm{PPT} / \mathrm{U} 3$ junction with even greater stacking rigidity at the $+1 \mathrm{a}$ site that prevents access of NMIA (analogous to our observations with the $-8 \mathrm{~T} / \mathrm{dF}$ 

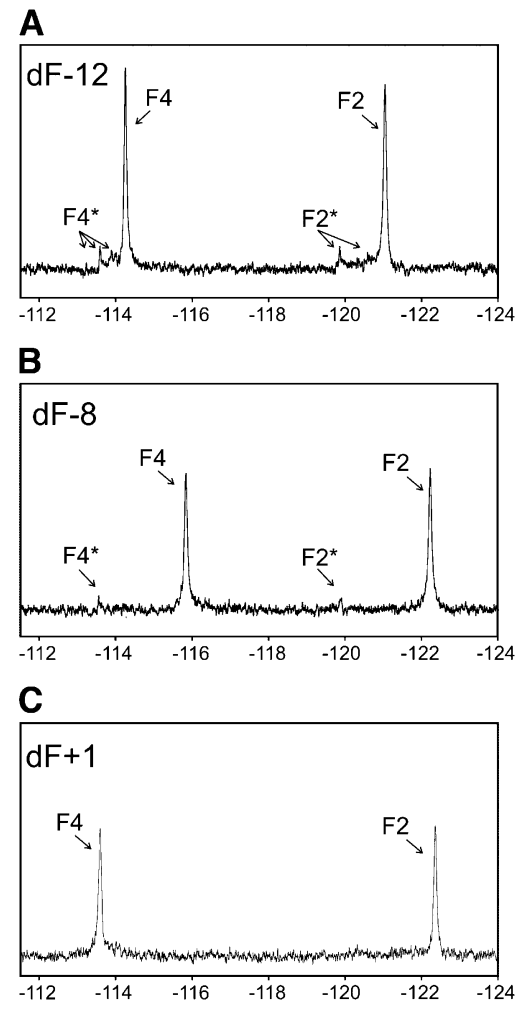

FIGURE 5. $1 \mathrm{D}{ }^{19}$ F NMR spectra of the HIV-1 PPT RNA/DNA hybrid with $\mathrm{T} / \mathrm{dF}$ substitutions at template positions $(A)-12,(B)-8$, and $(C)+1$. Spectra were acquired at $30^{\circ} \mathrm{C}$ with the fluorine carrier frequency set to $-118 \mathrm{ppm}$, sweep widths of $5646 \mathrm{~Hz}, 1024$ scans, and $4 \mathrm{~K}$ complex points. The $\mathrm{F} 2$ and $\mathrm{F} 4$ resonances for each $\mathrm{dF}$ base are labeled; asterisks indicate minor conformations.

hybrid), despite loss of hydrogen-bonding capacity. While the ${ }^{19} \mathrm{~F}$ spectrum and lack of acylation at $+1 \mathrm{a}$ in the $+1 \mathrm{dF}-$ substituted hybrid suggests rigidity at this position, the PPT/U3 junction is selectively targeted by the aminoglycoside neomycin $\mathrm{B}$, which has been shown to interact with motifs that disrupt regular double-helical patterns (Turner et al. 2008a). Similarly, chemical footprinting indicated that template $+1 \mathrm{~T}$ of the wild-type PPT RNA/DNA hybrid is sensitive to $\mathrm{KMnO}_{4}$ oxidation, suggesting that the duplex structure at the PPT/U3 junction deviates from canonical Watson-Crick geometry.

\section{NMIA sensitivity of the Ty3 retrotransposon PPT RNA/DNA hybrid}

Ty3 is a $S$. cerevisiae long terminal repeat-containing retrotransposon whose reverse transcription process shares many features with HIV-1. However, while Ty3 plus-strand DNA synthesis initiates from an RNA primer whose sequence, $5^{\prime}$-gagagagaggaa-3', differs significantly from the (rA:dT)n:(rC:dG)n architecture of retroviral PPTs (Rausch and Le Grice 2004), the Ty3 PPT is accurately processed, extended, and removed from nascent DNA by its cognate RT (Rausch et al. 2000). Since SHAMS highlighted structural anomalies in the wild-type HIV-1 PPT that were not detected by NMR (Yi-Brunozzi et al. 2008), the Ty3 PPT was investigated to determine if similar anomalies existed and if they could be modified by altering helix architecture.

A maximum of three NMIA adducts were observed on the wild-type Ty3 PPT RNA (data not shown). Gas-phase activation of the triply modified substrate indicated the terminal ribonucleotides $(-19 \mathrm{a},+5 \mathrm{u}$, and $+6 \mathrm{u})$ were NMIAsensitive, as well as the ribose of $+1 \mathrm{~g}$ (Fig. 6A). While modification of the terminal bases is attributed to fraying at the hybrid termini, selective modification of $+1 \mathrm{~g}$ is quite striking and consistent with previous NMR analysis that has shown an equilibrium between $\mathrm{C} 3{ }^{\prime}$-endo and $\mathrm{C} 2{ }^{\prime}$-endo sugar puckers at this position that may reflect conformational malleability at the PPT/U3 junction (Yi-Brunozzi et al. 2005).

Surprisingly, introducing a g:T wobble pair at position -6 renders the ribose of $-5 \mathrm{a},-6 \mathrm{~g}$, and $-7 \mathrm{a}$ NMIA-sensitive,
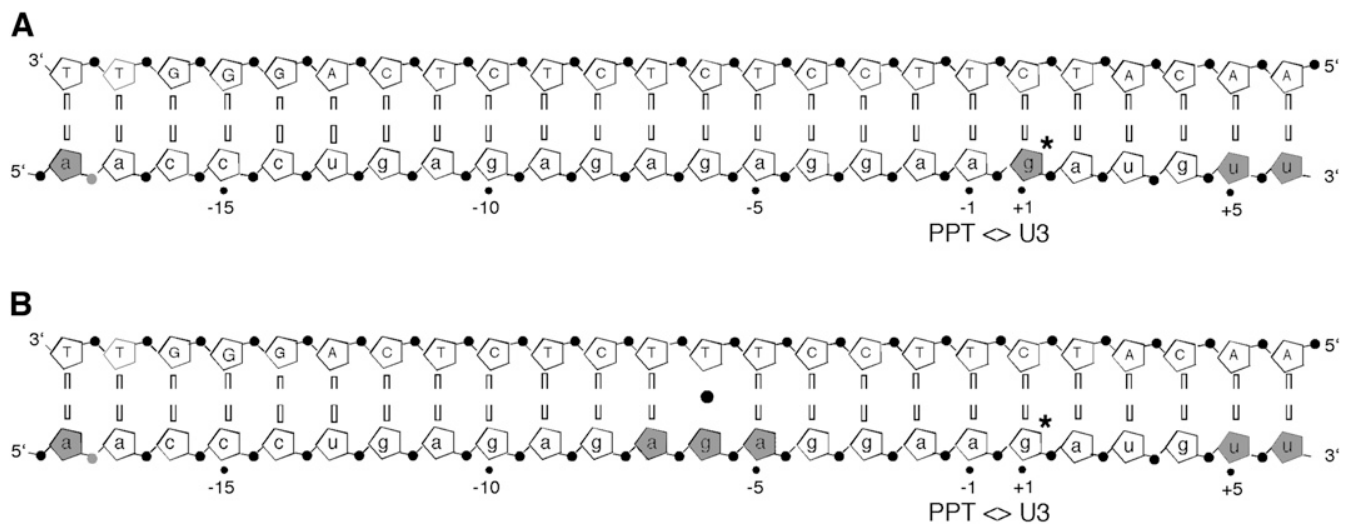

FIGURE 6. (A) Schematic of the wild-type S. cerevisiae Ty3 PPT RNA/DNA hybrid and $(B)$ a variant containing a $-6 \mathrm{G}$ :T mispair. Filled pentagons indicate NMIA sensitivity observed by mass spectrometry. The -6G:T mispair generates local sensitivity to NMIA at positions $-7,-6$, and -5 , but also eliminates sensitivity at $\left(^{*}\right)$ position +1 . 
while negating reactivity at position $+1 \mathrm{~g}$ (Fig. 6B). Our observations are quite striking as they show that this type of base-pair substitution, where pairing is presumably maintained, can significantly affect duplex conformation distal to the site; i.e., it is not restricted to adjacent backbone sugars. Thus, both the HIV-1 and Ty3 PPT hybrids appear to exhibit the potential for long-range structural coupling that might be exploited by their cognate RTs.

\section{DISCUSSION}

Our study of the HIV-1 and Ty3 PPTs clearly demonstrates that combining NMIA acylation with mass spectrometric characterization allows probing of short RNAs on which the primer extension step of SHAPE is impractical, or of substrates whose sequence or secondary structure prevents efficient primer extension. In support of these latter considerations, we note that, even in the absence of chemical modification, the PPT $(\mathrm{rG}) 6$ tract poses a significant barrier to reverse transcription of the RNA strand, resulting in prematurely terminated cDNA (JW Rausch and SFJ Le Grice, unpubl.). A particular advantage of the SHAMS method described here is that the ability to directly monitor the number and position of modified sites permits titration approaches to examine conformational effects induced by primary chemical modification. Based on these characteristics and the broad applicability of its analytical platform, the SHAMS approach should find general utility in structural biology. Our results obtained from PPT-containing hybrids substituted with the thymine isostere $\mathrm{dF}$ also provide a note of caution in interpreting the results of NMIA reactivity, which cannot be always linked solely to the absence of base-pairing. Conversely, the lack of $2^{\prime}-\mathrm{OH}$ modification should not be ascribed only to nucleotide participation in base-paired regions, but also to other effects constraining the ribose pucker conformation and to the steric protection induced by noncanonical basepairing, unusual base-stacking, cognate proteins, or other ligands, which is always possible with in vivo applications.

While NMR analysis suggests Watson-Crick base-pairing throughout the HIV-1 PPT in the absence of RT, with no evidence for unpaired or mispaired bases (Yi-Brunozzi et al. 2008), two acylation-sensitive internal positions $(-11 \mathrm{~g}$ and $-12 \mathrm{a})$ were detected by the SHAMS strategy. These new data are consistent with previous footprinting studies indicating that template thymines $-11 \mathrm{~T}$ and $-12 \mathrm{~T}$ are modestly responsive to $\mathrm{KMnO}_{4}$ oxidation in the absence of the retroviral polymerase (Kvaratskhelia et al. 2002). In both footprinting strategies, the reactive species represented a small fraction of the total population. Considering the results from the footprinting experiments, as well as the NMR data and crystal structure of the HIV-1 PPT•RT complex (Sarafianos et al. 2001), we propose that malleability of the HIV-1 PPT sequences around the $5^{\prime}$-aga- $3^{\prime}$ step between positions -12 and -10 is exploited to accommodate HIV-1 RT in a manner such that the active site of its C-terminal RNase $\mathrm{H}$ domain is correctly positioned over the scissile phosphodiester bond at the PPT-U3 junction (Fig. 1). It is interesting to note that, in this orientation, the malleable region would be immediately downstream from the DNA polymerase domain where crystallography indicates that the duplex is bent by $\sim 40^{\circ}$ (Jacobo-Molina et al. 1993; Huang et al. 1998; Sarafianos et al. 2001). Such malleability may manifest itself in transient unpairing or altered sugar pucker, thus rendering the ribose 2 -OH NMIA accessible. Sensitivity of this region to minor alterations in the local environment is exemplified by loss of acylation when template base $-8 \mathrm{~T}$ is replaced with $\mathrm{dF}$ (Fig. 4B). In addition, our recent analysis of RT/ PPT complexes by single-molecule spectroscopy has shown that minor alterations to the PPT can reverse enzyme orientation at the primer terminus (Abbondanzieri et al. 2008).

In summary, we demonstrate that chemical acylation can be directly applied to probe conformational features of the RNA of RNA/DNA hybrids, with emphasis here on the plus-strand, PPT RNA primers of a human retrovirus (HIV-1) and S. cerevisiae LTR-containing retrotransposon (Ty3). Although not widespread, there are several examples of RNA/DNA hybrids with biological significance, including their involvement in immunoglobin class switching (Tracy et al. 2000), human cytomegalovirus virus lytic DNA replication (Pari 2008), and ColE1 plasmid replication (Santamaria et al. 1998). The ease with which NMIA treatment can be combined with mass spectrometry should allow a more detailed analysis of these duplexes, either alone or in conjunction with their protein partners. Finally, while our SHAMS approach has broad utility in detecting subtle alterations in nucleic acid geometry, the HIV PPT represents a system in which interpreting these changes benefits from the availability of X-ray crystal structures, high-resolution NMR analysis, and biochemical probing of analog-substituted RNA/DNA hybrids. Complementing chemical probing with data obtained using other biochemical and biophysical techniques will clearly be required to interpret the biological significance of such subtle structural modifications in other biological systems.

\section{MATERIALS AND METHODS}

\section{Nucleic acid substrates}

RNA and DNA phosphoramidites, including 2,4-difluoro-5methylbenzene, were purchased from Glen Research Corp. Oligonucleotides were synthesized at a $1-\mu \mathrm{mol}$ scale using standard phosphoramidite chemistry on a PE Biosystems Expedite 8909 nucleic acid synthesizer and purified by preparative polyacrylamide gel electrophoresis. RNA and DNA single strands were annealed by mixing equimolar amounts, heating the solution for 3 min to $90^{\circ} \mathrm{C}$, and allowing the samples to cool slowly to form the 
duplex. Duplex concentrations were determined from absorbance at $260 \mathrm{~nm}$ (CARY300 UV-Vis Spectrophotometer), using a molar extinction coefficient that is equal to the sum of the ribo- and deoxyribonucleotides $\left(384,900 \mathrm{~cm}^{-1} \mathrm{M}^{-1}\right.$ and $379,700 \mathrm{~cm}^{-1} \mathrm{M}^{-1}$ for Ty3 and HIV-1 PPT duplexes, respectively). Final duplex concentrations for NMR measurements were typically $1.0 \mathrm{mM}$.

\section{Nucleic acid substrates}

Through titration and time-course experiments, chemical acylation reactions were optimized to avoid possible substrate distortion, as described in the text. As a result, the data discussed in Results and Discussion required reacting $50 \mu \mathrm{M}$ RNA/DNA hybrid with up to $500 \mu \mathrm{M}$ NMIA (final solution concentrations) for $50 \mathrm{~min}$ at $37^{\circ} \mathrm{C}$. Increasing the final probe concentration to greater than 10 -fold excess induced multiple nonspecific probing events.

\section{Mass spectrometry}

MS analyses were performed on a Bruker Daltonics Apex III FTICR mass spectrometer equipped with a 7T actively shielded superconducting magnet and a nano-ESI source constructed in house. Reaction mixtures were quenched via ethanol precipitation. The pellet was resuspended in $5 \mathrm{mM}$ ammonium acetate and warmed to $80^{\circ} \mathrm{C}$ to reduce the hybridization and allow for more facile data analysis. Typically, $5 \mu \mathrm{L}$ of the warm analyte solutions was loaded into the nano-ESI needle, and a spray voltage of $<1 \mathrm{kV}$ was applied to the solution through a stainless steel wire inserted in the rear end of the needle. No solvent pumps were necessary, as the solution flow rate was dictated by the applied voltage and size of the nano-ESI needle tip (typically $\sim 1-2 \mu \mathrm{m}$ ). Spectra were acquired in negative ionization mode and processed using ApexControl 2.0 (Bruker Daltonics). Scans were completed in broadband mode that allowed for a typical 150,000 resolving power at $\mathrm{m} / z$ 2000. Spectra were externally calibrated using a 1 $\mathrm{mg} / \mathrm{mL}$ solution of CsI, which produced a series of peaks throughout the mass range of $1000-6000 \mathrm{~m} / z$ and provided a typical mass accuracy of 20 ppm or better across the range. Each analysis was performed a minimum of three times.

In tandem MS experiments, the precursor ion of interest was isolated in the FTICR cell using correlated rf sweeps (de Koning et al. 1997), then activated through SORI-CID (Gauthier et al. 1991). Frequency offsets below and above the resonant frequency of the precursor ion were sampled to avoid possible "blind spots" in the product spectra. Best results were achieved with irradiation frequencies 600 to $2000 \mathrm{~Hz}$ below that of the precursor ion. Typical activation regimes were reached by applying off-resonant pulses for $250 \mathrm{msec}$, using $26-31 \mathrm{~dB}$ attenuation of the maximum power output allowed by the hardware. Argon was used as the collision gas in pulsed bursts of 100-250 msec, which resulted in momentary increases from $1 \times 10^{-11}$ to $1 \times 10^{-7}$ mbar of the background pressure measured by the instrument gauge located underneath the ion optics. No attempt was made to determine the actual pressure within the FTICR cell.

\section{NMR analysis}

NMR samples were examined in $80 \mathrm{mM} \mathrm{NaCl}$ and $10 \mathrm{mM}$ phosphate $\left(\mathrm{pH} 7.0\right.$ ) that were dissolved in $90 \% \mathrm{H}_{2} \mathrm{O} / 10 \% \mathrm{D}_{2} \mathrm{O}$ or
99.96\% $\mathrm{D}_{2} \mathrm{O}$. One-dimensional ${ }^{19} \mathrm{~F}$ experiments were performed on a Bruker Biospin DRX500 spectrometer equipped with a direct-detect ${ }^{19} \mathrm{~F}$ probe. Two-dimensional spectra were collected on a Bruker Biospin DMX600 equipped with either a TXI HCN probe or a $\mathrm{PH}$ SEF 600SB F-H-D-05 Z probe. All spectra were processed with Topspin and analyzed using Sparky or Topspin on a PC/Linux workstation. Assignments were made using DQFCOSY, NOESY, TOCSY, ${ }^{1} \mathrm{H}-{ }^{13} \mathrm{C}$ (natural abundance) HMQC, ${ }^{1} \mathrm{H}-{ }^{19} \mathrm{~F}$ HSQC, and ${ }^{1} \mathrm{H}-{ }^{19} \mathrm{~F}$ NOESY. Other experimental details and acquisition parameters are given in the Figure 5 legend.

\section{ACKNOWLEDGMENTS}

This research was funded by National Institutes of Health grants GM59107 (to J.P.M.) and GM643208 (to D.F.), and grant CHE0439067 from the National Science Foundation (to D.F.). S.F.J.L.G. was supported by the intramural research program of the Center for Cancer Research, National Cancer Institute, National Institutes of Health.

Received February 23, 2009; accepted April 29, 2009.

\section{REFERENCES}

Abbondanzieri EA, Bokinsky G, Rausch JW, Zhang JX, Le Grice SF, Zhuang X. 2008. Dynamic binding orientations direct activity of HIV reverse transcriptase. Nature 453: 184-189.

Badorrek CS, Weeks KM. 2006. Architecture of a gamma retroviral genomic RNA dimer. Biochemistry 45: 12664-12672.

Cerny RL, Tomer KB, Gross ML, Grotjahn L. 1987. Fast atom bombardment combined with tandem mass spectrometry for determining structures of small oligonucleotides. Anal Biochem 165: $175-182$.

Comisarow MB, Marshall AG. 1974. Fourier transform ion cyclotron resonance. Chem Phys Lett 25: 282-283.

de Koning LJ, Nibbering NMM, van Orden SL, Laukien FH. 1997. Mass selection of ions in a Fourier transform ion cyclotron resonance trap using correlated harmonic excitation fields (CHEF). Int J Mass Spectrom Ion Process 165/166: 209-219.

Ehresmann C, Baudin F, Mougel M, Romby P, Ebel JP, Ehresmann B. 1987. Probing the structure of RNAs in solution. Nucleic Acids Res 15: 9109-9128.

Gauthier JW, Trautman TR, Jacobson DB. 1991. Sustained offresonance irradiation for collision-activated dissociation involving Fourier transform mass spectrometry. Collision-activated dissociation technique that emulates infrared multiphoton dissociation. Anal Chim Acta 246: 211-225.

Gherghe CM, Mortimer SA, Krahn JM, Thompson NL, Weeks KM. 2008. Slow conformational dynamics at $\mathrm{C} 2$ '-endo nucleotides in RNA. J Am Chem Soc 130: 8884-8885.

Guckian KM, Krugh TR, Kool ET. 1998. Solution structure of a DNA duplex containing a replicable difluorotoluene-adenine pair. Nat Struct Biol 5: 954-959.

Hendrickson CL, Emmett MR, Marshall AG. 1999. Electrospray ionization Fourier transform ion cyclotron resonance mass spectrometry. Annu Rev Phys Chem 50: 517-536.

Huang H, Chopra R, Verdine GL, Harrison SC. 1998. Structure of a covalently trapped catalytic complex of HIV-1 reverse transcriptase: implications for drug resistance. Science 282: 16691675.

Jacobo-Molina A, Ding J, Nanni RG, Clark AD Jr, Lu X, Tantillo C, Williams RL, Kamer G, Ferris AL, Clark P, et al. 1993. Crystal structure of human immunodeficiency virus type 1 reverse transcriptase complexed with double-stranded DNA at $3.0 \AA$ resolution shows bent DNA. Proc Natl Acad Sci 90: 6320-6324. 
Kellersberger KA, Yu E, Kruppa GH, Young MM, Fabris D. 2004. Top-down characterization of nucleic acids modified by structural probes using high-resolution tandem mass spectrometry and automated data interpretation. Anal Chem 76: 2438-2445.

Kirpekar F, Krogh TN. 2001. RNA fragmentation studied in a matrixassisted laser desorption/ionisation tandem quadrupole/orthogonal time-of-flight mass spectrometer. Rapid Commun Mass Spectrom 15: 8-14.

Klarmann GJ, Hawkins ME, Le Grice SF. 2002. Uncovering the complexities of retroviral ribonuclease $\mathrm{H}$ reveals its potential as a therapeutic target. AIDS Rev 4: 183-194.

Kopka ML, Lavelle L, Han GW, Ng HL, Dickerson RE. 2003. An unusual sugar conformation in the structure of an RNA/DNA decamer of the polypurine tract may affect recognition by RNase H. J Mol Biol 334: 653-665.

Kvaratskhelia M, Budihas SR, Le Grice SF. 2002. Pre-existing distortions in nucleic acid structure aid polypurine tract selection by HIV-1 reverse transcriptase. J Biol Chem 277: 16689-16696.

McLuckey SA, Habibi-Goudarzi S. 1993. Decompositions of multiply charged oligonucleotide anions. J Am Chem Soc 115: 1208512095 .

Nordhoff E, Kirpekar F, Roepstorff P. 1996. Mass spectrometry of nucleic acids. Mass Spectrom Rev 15: 67-138.

Pari GS. 2008. Nuts and bolts of human cytomegalovirus lytic DNA replication. Curr Top Microbiol Immunol 325: 153-166.

Rausch JW, Le Grice SF. 1997. Substituting a conserved residue of the ribonuclease $\mathrm{H}$ domain alters substrate hydrolysis by retroviral reverse transcriptase. J Biol Chem 272: 8602-8610.

Rausch JW, Le Grice SF. 2004. 'Binding, bending, and bonding': Polypurine tract-primed initiation of plus-strand DNA synthesis in human immunodeficiency virus. Int J Biochem Cell Biol 36: 1752-1766.

Rausch JW, Grice MK, Henrietta M, Nymark M, Miller JT, Le Grice SF. 2000. Interaction of p55 reverse transcriptase from the Saccharomyces cerevisiae retrotransposon Ty3 with conformationally distinct nucleic acid duplexes. J Biol Chem 275: 1387913887.

Rausch JW, Qu J, Yi-Brunozzi HY, Kool ET, Le Grice SF. 2003. Hydrolysis of RNA/DNA hybrids containing nonpolar pyrimidine isosteres defines regions essential for HIV type 1 polypurine tract selection. Proc Natl Acad Sci 100: 11279-11284.

Santamaria D, de la Cueva G, Martinez-Robles ML, Krimer DB, Hernandez P, Schvartzman JB. 1998. DnaB helicase is unable to dissociate RNA-DNA hybrids. Its implication in the polar pausing of replication forks at ColE1 origins. J Biol Chem 273: 3338633396.

Sarafianos SG, Das K, Tantillo C, Clark AD Jr, Ding J, Whitcomb JM, Boyer PL, Hughes SH, Arnold E. 2001. Crystal structure of HIV-1 reverse transcriptase in complex with a polypurine tract RNA: DNA. EMBO J 20: 1449-1461.
Tracy RB, Hsieh CL, Lieber MR. 2000. Stable RNA/DNA hybrids in the mammalian genome: Inducible intermediates in immunoglobulin class switch recombination. Science 288: 1058-1061.

Tromp JM, Schurch S. 2005. Gas-phase dissociation of oligoribonucleotides and their analogs studied by electrospray ionization tandem mass spectrometry. J Am Soc Mass Spectrom 16: 1262-1268.

Turner KB, Brinson RG, Yi-Brunozzi HY, Rausch JW, Miller JT, Le Grice SF, Marino JP, Fabris D. 2008a. Structural probing of the HIV-1 polypurine tract RNA:DNA hybrid using classic nucleic acid ligands. Nucleic Acids Res 36: 2799-2810.

Turner KB, Monti S, Fabris D. 2008b. Like polarity ion/ion reactions enable the investigation of specific metal interactions in nucleic acids and their non-covalent assemblies. J Am Chem Soc 30: 13353-13363.

Vicens Q, Gooding AR, Laederach A, Cech TR. 2007. Local RNA structural changes induced by crystallization are revealed by SHAPE. RNA 13: 536-548.

Wang B, Wilkinson KA, Weeks KM. 2008. Complex ligand-induced conformational changes in tRNA ${ }^{\text {(Asp) }}$ revealed by single-nucleotide resolution SHAPE chemistry. Biochemistry 47: 3454-3461.

Wilkinson KA, Merino EJ, Weeks KM. 2006. Selective 2'-hydroxyl acylation analyzed by primer extension (SHAPE): Quantitative RNA structure analysis at single nucleotide resolution. Nat Protocols 1: 1610-1616.

Wilkinson KA, Gorelick RJ, Vasa SM, Guex N, Rein A, Mathews DH, Giddings MC, Weeks KM. 2008. High-throughput SHAPE analysis reveals structures in HIV-1 genomic RNA strongly conserved across distinct biological states. PLoS Biol 6: e96. doi: 10.1371/ journal.pbio.0060096.

Yi-Brunozzi HY, Brabazon DM, Lener D, Le Grice SF, Marino JP. 2005. A ribose sugar conformational switch in the LTR-retrotransposon Ty3 polypurine tract-containing RNA/DNA hybrid. $J$ Am Chem Soc 127: 16344-16345.

Yi-Brunozzi HY, Brinson RG, Brabazon DM, Lener D, Le Grice SF, Marino JP. 2008. High-resolution NMR analysis of the conformations of native and base analog substituted retroviral and LTRretrotransposon PPT primers. Chem Biol 15: 254-262.

Yu E, Fabris D. 2003. Direct probing of RNA structures and RNAprotein interactions in the HIV-1 packaging signal by chemical modification and electrospray ionization Fourier transform mass spectrometry. J Mol Biol 330: 211-223.

Yu ET, Fabris D. 2004. Toward multiplexing the application of solvent accessibility probes for the investigation of RNA three-dimensional structures by electrospray ionization-Fourier transform mass spectrometry. Anal Biochem 344: 356-366.

Yu ET, Zhang Q, Fabris D. 2005. Untying the FIV frameshifting pseudoknot structure by MS3D. J Mol Biol 345: 69-80.

Yu ET, Hawkins AE, Eaton J, Fabris D. 2008. MS3D structural elucidation of the HIV-1 packaging signal. Proc Natl Acad Sci 105: $12248-12253$. 

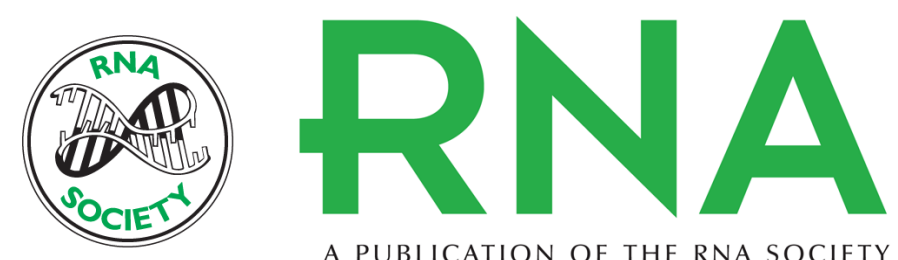

A PUBLICATION OF THE RNA SOCIETY

\section{SHAMS: Combining chemical modification of RNA with mass spectrometry to examine polypurine tract-containing RNA/DNA hybrids}

Kevin B. Turner, Hye Young Yi-Brunozzi, Robert G. Brinson, et al.

RNA 2009 15: 1605-1613 originally published online June 17, 2009

Access the most recent version at doi:10.1261/rna.1615409

\section{References This article cites 40 articles, 11 of which can be accessed free at:} http://rnajournal.cshlp.org/content/15/8/1605.full.html\#ref-list-1

\section{License}

Email Alerting Receive free email alerts when new articles cite this article - sign up in the box at the Service top right corner of the article or click here. 